\title{
Surgical management of traumatic ventro-abdominal hernia in a crossbred cattle
}

\author{
M. Lal ${ }^{*}$, Kuldeep ${ }^{1}$, S. Pandey ${ }^{2}$, D. Das ${ }^{2}$ and C. Deka ${ }^{3}$ \\ ${ }^{1}$ Department of Veterinary Surgery and Radiology, MJF College of Veterinary and Animal Science, \\ Chomu, Jaipur- 303 702, Rajasthan, India; ${ }^{2}$ Department of Veterinary Medicine, MJF College of \\ Veterinary and Animal Science, Chomu, Jaipur - 303 702, Rajasthan, India; ${ }^{3}$ Department of Veterinary \\ Medicine, College of Veterinary Sciences and Animal Husbandry, CAU, Selesih, Aizawl - 796014 , \\ Mizoram, India
}

\begin{abstract}
A five-year-old HF crossbred cattle was presented to the Veterinary Clinical Complex, MJF College of Veterinary and Animal Science, Chomu, Jaipur, with a large external swelling in the ventral abdominal region for a week. The clinical examination revealed large soft reducible swelling and a hernial ring on the ventro-abdominal region, and the case was confirmed as a hernia. The animal was restrained in left lateral recumbency under sedation with xylazine and infiltration of local anaesthetic at the site. Surgical herniorrhaphy was done by using synthetic non-absorbable polyester ribbon in overlapping mattress suture techniques. After complete follow-up, the animal was recovered uneventfully.
\end{abstract}

Key words: Cattle, Herniorrhaphy, Reducible, Ventro-abdominal hernia

\section{Highlights}

- The case was diagnosed as a traumatic ventral abdominal hernia.

- Sedation was achieved with xylazine followed by local infiltration at the operative site.

- Sterilized synthetic and non-absorbable polyester ribbons were used during herniorrhaphy.

- Recovery was uneventful after repositioning the herniated mass into its normal anatomical position.

Ventral abdominal hernia means a protrusion of the viscera, especially intestines, through a tear in any part of the abdominal wall other than a natural orifice, and it is ventral to the stifle skin fold (Farman et al., 2018). It most commonly occurs due to trauma by external forces or trauma to the abdominal wall (Radhakrishnan et al., 1993) and automobile accident (Vijayanand et al., 2009). The hernia size varies depending on the extent of the hernial defect and the amount of abdominal contents contained within it. The diagnosis was made from the history and by palpation of the swollen region and confirmation of reducible hernia done through the contents of the hernia and hernial ring (Amare and Haben, 2020), but in the case of irreducible hernias, radiographic examination is needed to reflect the lack of abdominal wall continuity. The only effective treatment for an abdominal hernia is surgery to restore the integrity of the abdominal wall and prevent incarceration and strangulation of herniated contents (Jahromi et al., 2009). Application of bandage, clamps, or ligatures may be helpful in a few cases where the hernial ring is small (Villar et al., 2011). Herniorrhaphy is useful in case of large hernial opening, but extensive ventral abdominal hernia may require hernioplasty (Villar et al., 2011), and complex abdominal wall and hernia repair by use a combination of primary and mesh techniques (Whitfield-Cargile et al., 2011).

Hence, the present study describes the successful management of a traumatic ventroabdominal hernia in a Holstein Frisian cross cattle. 
A five-year-old Holstein Frisian cross cattle was presented to the Veterinary Clinical Complex, MJF College of Veterinary and Animal Science, Chomu, Jaipur, with a large external swelling on the ventral abdominal region for a week. According to the owner, the animal was injured one week ago before being presented to the clinics. Anamnesis suggested that the cattle lost its appetite, and swelling at the ventral abdomen was increasing with its age for one week. Clinical examination revealed the hernial content firm, nonpainful and reducible within the hernial ring. Clinical parameters like heart rate, respiratory rate and rectal temperature were within the normal physiological limits. The presented case was diagnosed as a ventral abdominal hernia and suggested surgery to correct the condition.

The water was withheld for 12 hours and feed for 24 hours before surgery. The animal was restrained in left lateral recumbency, and the area was prepared aseptically for surgery. The animal was sedated with xylazine hydrochloride@ @ $0.05 \mathrm{mg} / \mathrm{kg} \quad$ BW intramuscularly, followed by local infiltration at the site using $2 \%$ lignocaine hydrochloride with a dose rate $1 \mathrm{~mL} / \mathrm{cm}$ area. A longitudinal incision was given in the middle of the swelling, separating the skin and subcutaneous tissue by blunt dissection to reach the hernial sac. After opening the sac, $20-25 \mathrm{~cm}$ hernial ring was exposed. After skin and subcutaneous tissue dissection, the condition of the hernial sac and ring was examined carefully to confirm the presence or absence of adhesions of the abdominal organs. The herniated viscera were repositioned in the abdominal cavity by manual taxis. Then herniorrhaphy was achieved. The herniorrhaphy was done using sterilized synthetic and non-absorbable polyester ribbon in an overlapping mattress suture pattern (Fig. 2). Abdominal muscles and subcutaneous tissue were apposed with simple continuous suture using catgut size no. 2 , and skin edges were apposed with cross mattress suture using silk size no. 2. Finally, stay sutures were applied on the skin to hold the gauge over the incision line to prevent infection. A supportive coarse cotton tape (Niwar) was placed around the abdomen to relieve tension on the healing tissue (Fig. 1).

Post-operatively, administered ceftriaxone and tazobactum @ $10 \mathrm{mg} / \mathrm{kg}$ BW intramuscularly twice daily for 5 days and meloxicam@0.2 mg/kg BW intramuscularly once daily for 3 days along with regular antiseptic dressing using povidone-iodine for 12 days. The sutures were removed on the $12^{\text {th }}$ post-operative day and advised the owner that the animal should be fed on soft food minimum for 2 weeks after the operation to minimize pressure on the site of the incision. The healing process was clinically evaluated, and the surgical wound was completely healed after 12 days of operation (Fig. 2).

A ventral abdominal hernia is commonly acquired in nature and caused by trauma (kicks, horn thrust, violent contact with a blunt object and sudden jump) that results in rupture of the abdominal muscles in large animals. A ventral hernia is commonly seen in the ventral abdominal wall near the midline. The size of the hernial opening varies in diameter, and the nature of hernial contents depends on the site of the herniation (Fossum, 2013). In the case of a reducible hernia, the contents went back to the abdominal cavity, and the hernial ring became evident (Salim et al., 2015). In the case to make of herniorrhaphy, xylazine hydrochloride can be used as a better sedative, while $0.5 \%$ bupivacaine hydrochloride can be used as a local analgesic for a longer duration of action (Sarker et al., 2012). There are several techniques to treat a hernia, surgical and nonsurgical, depending on the size and the type of a hernia. In the case of surgical techniques, there are two types of open and closed surgical operations (Farman et al., 2018). Application of bandage, clamps or ligatures may be helpful in a few cases where the hernia ring is small. Surgical intervention is useful in the case of large hernial openings (Abdin-bey and Ramadan, 2001). In case of simple and small hernia in size, they are treated by suturing the edges of the ring with absorbable suture (Farman et al., 2018) or non-absorbable nylon ribbon (Kaswan 
et al., 2014), but in case of large hernia nonabsorbable mesh to support the muscles must be used. Mesh may cause irritation in its site, cause post-operative pain, and delay healing time. Polypropylene mesh is one of the most commonly used prosthetic materials for large ventral hernia repair in large animals (Finan et al., 2009). The amount of feed should be reduced to half for about a week after surgery, and a supportive bandage may be placed around the abdomen to relieve tension on the healing tissues (Tyagi and Singh, 1995). Herniorrhaphy was performed in the present case using synthetic polyester ribbon in overlapping mattress patterns without any complication.

\section{REFERENCES}

Abdin-bey MR and Ramadan RO, 2001. Retrospective study of hernias in goats. Sci J King Faisal Univ, 2(1): 77-88

Amare E and Haben F, 2020. Hernias in farm animals and its management technique - A review. IJCMCR, 4(4): 001, doi: 10.46998/ IJCMCR.2020.04.000091

Farman RH, Al-Husseiny SH and Abd Al-Ameer AN, 2018. Surgical treatment of hernia in cattle: A review. Al-Qadisiyah J Vety Med Sci, 17(2): 6168, doi: 10.29079/vol17iss2art506

Finan KR, Kilgore ML and Hawn MT, 2009. Open suture versus mesh repair of primary incisional hernias: A cost-utility analysis. Hernia, 13(2): 173-182, doi: 10.1007/s10029-008-0462-1

Fossum TW, 2013. Small Animal Surgery TextbookE-Book. Elsevier Health Sciences

Jahromi AR, Nazhvani SD, Gandmani MJ and Mehrshad S, 2009. Concurrent bilateral inguinal and umbilical hernias in a bitch - A case report. Vet Arhiv, 79(5): 517-522

Kaswan BL, Purohit NR and Gahlot TK, 2014. Surgical management of abdominal hernia in a camel (Camelus dromedaries). Camel: Int J Vety Sci, 2(2): 15-18, doi: 10.5958/2321709X.2014.00003.7

Radhakrishnan C, Balasubramanian S and Thilagar S,
Conflict of interest: Authors have no conflict of interest in this study.

Author's contribution: ML, KD \& DD: Performed the clinical and treatment part of the study and provided the treatment feedback; SP \& CD: Drafted the manuscript with subsequent revisions; DD finalized the manuscript and sent it for publication. All authors read and approved the final manuscript.

\section{ACKNOWLEDGEMENT}

The authors are grateful to the Hon'ble Dean of MJF CVAS, Chomu, Jaipur, for providing the necessary facilities for conducting this work.

1993. Repair of ventral metrocele (gravid) in a goat. Vet Rec, 132(4): 92, doi: 10.1136/ vr.132.4.92

Salim MD, Hashi MA, Juyena NS, Arafat YA, Dey RKet al., 2015. Prevalence of hernia and evaluation of herniorrhaphy in calves. Int J Nat Soc Sci, 2(4): 35-43

Sarker NC, Hashim MA and Ray BP, 2012. Effect of Sedative techniques on physiological parameters of calves at (minor surgical procedures) $\mathrm{L}$ or herniorrhaphy. J Agric Sci, 7(2): 66-75, doi: 10.4038/jas.v7i2.4406

Tyagi RPS and Singh J, 1995. Ruminant Surgery. CBS Publication

Vijayanand V, Gokulakrishnan M, Rajasundaram RC and Thirunavukkarasu PS, 2009. Ventral hernia (hysterocele-gravid) in a goat - A case report. Ind J Anim Res, 43(2): 148-150

Villar JM, Corbera JA and Spinella G, 2011. Doublelayer mesh hernioplasty for repairing umbilical hernias in 10 goats. Turkish J Vet Anim Sci, 35(2): 131-135, doi: 10.3906/vet-1006-365

Whitfield Cargile CM, Rakestraw PC, Hardy J, Cohen ND and Davis BE, 2011. Comparison of primary closure of incisional hernias in horses with and without the use of prosthetic mesh support. Equine Vet J, 43(39): 69-75, doi: 10.1111/j.20423306.2011.00377.x

Received - 18.11.2021, Accepted - 06.02.2022, Published - 17.02.2022 (Online), 01.06.2022 (Print)

Section Editor: Prof. S. K. Nandi, Associate Editor 